\title{
Measuring two-qubit gates
}

\author{
Andrew G. White and Alexei Gilchrist \\ Department of Physics and Centre for Quantum Computer Technology, University of Queensland, Brisbane QLD \\ 4072, Australia \\ Geoffrey J. Pryde \\ Department of Physics and Centre for Quantum Computer Technology, University of Queensland, Brisbane QLD \\ 4072, Australia, and Centre for Quantum Computer Technology and Centre for Quantum Dynamics, School \\ of Science, Griffith University, Brisbane QLD 4111, Australia \\ Jeremy L. O'Brien \\ Department of Physics and Centre for Quantum Computer Technology, University of Queensland, Brisbane QLD \\ 4072, Australia, and H. H. Wills Physics Laboratory and Department of Electrical and Electronic Engineering,
} University of Bristol, Bristol BS8 1UB, UK

\section{Michael J. Bremner}

Department of Physics and Centre for Quantum Computer Technology, University of Queensland, Brisbane QLD 4072, Australia, and Institüt für Theretische Physik, Universität Innsbruck, 6020 Innsbruck, Austria

\section{Nathan K. Langford}

Department of Physics and Centre for Quantum Computer Technology, University of Queensland, Brisbane QLD 4072, Australia

Received September 19, 2006; accepted October 4, 2006;

posted October 19, 2006 (Doc. ID 75158); published January 26, 2007

Accurate characterization of two-qubit gates will be critical for any realization of quantum computation. We discuss a range of measurements for characterizing two-qubit gates. These measures are architectureindependent and span a range of complexity from simple measurement routines to full quantum-state and process tomography. Simple indicative measures, which flag but do not quantify gate operation in the quantum regime, include the fringe visibility, parity, Bell-state fidelity, and entanglement witnesses. Quantitative measures of gate output states include linear entropy and tangle; measures of, and error bounds to, whole-gate operation are provided by metrics such as process fidelity, process distance, and average gate fidelity. We discuss which measures are appropriate, given the stage of development of the gate, and highlight connections between them. (C) 2007 Optical Society of America

OCIS codes: 270.0270.

\section{INTRODUCTION}

The quest to realize a scaleable quantum computing architecture is one of the key aspects of current quantum information science. Many paradigms require at least these two elements: single-qubit arbitrary rotation gates and two-qubit entangling gates, such as a controlled-NOT (CNOT) gate, ${ }^{1}$ although more generally any gate that nonmaximally entangles two qubits can be used. ${ }^{2,3}$ A wide variety of experimental architectures are currently under consideration for realizing two-qubit gates, ${ }^{4}$ such as electronic and nuclear spin- $\frac{1}{2}$ systems; atomic, ionic, and phononic simple harmonic oscillators; superconducting systems; and optical systems.

Common to all these architectures is the need for an accurate characterization of the single-and two-qubit gates. Characterizing a single-qubit gate operation is straightforward; characterizing a two-qubit gate is more complex, as the gate modifies the joint output state and so, for example, can entangle or disentangle the input state. Further, it is desirable to diagnose, and if possible correct, error behaviors introduced by a real gate, such as phase or bit-flip errors, which can induce the wrong amount or type of entanglement or decoherence.

To date, there have been a wide variety of measures used to gauge the quality of two-qubit gates. These measures are often not comparable, making it difficult to sensibly compare gate operation in one architecture with that in another. We establish an architectureindependent measurement standard for two-qubit gates. In this paper we categorize measures into three types:

- Indicative measures flag, but do not quantify, entangled state production.

- State measures quantify output state properties. 
- Process measures quantify gate operation.

We examine a range of measures in the extant literature highlighting the conditions where they are best applied-using a CNOT gate to illustrate the principles. We conclude that only process measures allow definitive and quantitative statements about two-qubit gate operation, and these measures must be used for benchmarking gates against desired standards. ${ }^{4}$

\section{INDICATIVE MEASURES}

\section{A. Truth Table}

The first measure of any gate is its truth table. Without loss of generality, we consider the reversible exclusive-OR (XOR) gate-the classical two-bit CNOT gate, where a control bit flips the state of a target bit. As shown in Table 1, there are only four possible input, and therefore output, states. The simplest characterization of an unknown twobit gate is a straightforward matter of entering each possible input and measuring the output state. A more complete characterization, which allows measurement of error probabilities, is to measure the probability of each of the four possible output states for each of the four inputs, yielding a truth table, $M_{0}$, as shown in Table 2 . One measure of the overlap between a measured truth table, $M_{\text {exp }}$, and the ideal truth table is given by the average of the logical basis fidelities, the inquisition, $\mathcal{I}$ $=\operatorname{Tr}\left(M_{\text {exp }} M_{0}^{T}\right) / \operatorname{Tr}\left(M_{0} M_{0}^{T}\right)$. To date, ionic and photonic CNOT gates have achieved inquisitions of $73 \%$ (see Ref. 5) and $94 \pm 3 \%$ (see Ref. 6), respectively.

For quantum gates, the input qubits can exist in an arbitrary complex superposition of a classical bit, i.e. $|c\rangle_{\text {in }}$ $=\alpha|0\rangle+\beta|1\rangle$ and $|t\rangle_{\text {in }}=\gamma|0\rangle+\delta|1\rangle$, where $|\alpha|^{2}+|\beta|^{2}=1$ and $|\gamma|^{2}+|\delta|^{2}=1$. The gate has infinitely many possible inputs, and characterization is not a simple matter of exhausting all possible inputs. The difference to the classical gate is deeper than this; applying Table 1 , we see that the output state is $|c t\rangle_{\text {out }}=\alpha \gamma|00\rangle+\alpha \delta|01\rangle+\beta \delta|10\rangle+\beta \gamma|11\rangle$. For certain

Table 1. Input-Output Values of a Reversible xoR Gate $^{a}$

\begin{tabular}{|c|c|}
\hline$(\mathrm{ct})_{i n}$ & (ct $)_{o u t}$ \\
\hline 00 & 00 \\
\hline 01 & 01 \\
\hline 10 & 11 \\
\hline 11 & 10 \\
\hline
\end{tabular}

${ }^{a} c$ and $t$ refer to control and target, respectively.

Table 2. Truth Table of a Reversible xor Gate ${ }^{a}$

\begin{tabular}{ccccc}
\hline & \multicolumn{4}{c}{ Output Qubits } \\
\cline { 2 - 5 } Input & $(00)_{o}$ & $(01)_{o}$ & $(10)_{o}$ & $(11)_{o}$ \\
\hline$(00)_{i}$ & 1 & 0 & 0 & 0 \\
$(01)_{i}$ & 0 & 1 & 0 & 0 \\
$(10)_{i}$ & 0 & 0 & 0 & 1 \\
$(11)_{i}$ & 0 & 0 & 1 & 0 \\
\hline
\end{tabular}

${ }^{a}$ Values are the probability of achieving the selected output state, $(c t)_{o}$, for a given input state, $(c t)_{i}$ coefficient values, e.g., $\delta=0, \alpha, \beta \neq 0$, the output state is not just the product of the input states-it is entangled, with correlations between the output qubits that cannot be replicated by classical physical models.

\section{B. Visibility and Parity}

Clearly, a signature of quantum gate operation is the generation of entangled output states-thus it is necessary to identify, and preferably quantify, these outputs. A beginning point is to measure a series of correlations, or jointmeasurement probabilities, between the control and target arms, with the aim of identifying uniquely quantum correlations. Consider, for example, the case of a controlsuperposition input, where we rewrite the inputs as $|\psi\rangle_{c}$ $=(|0\rangle+\varepsilon|1\rangle) / \sqrt{1+|\varepsilon|^{2}}$ and $|\psi\rangle_{t}=|0\rangle$. A CNOT gate outputs the entangled state $|\psi\rangle_{\text {out }}=(|00\rangle+\varepsilon|11\rangle) / \sqrt{1+|\varepsilon|^{2}}$ (where $\varepsilon$ is a $c$-number and $|\varepsilon|=1$ gives a maximally entangled state). Note that for a target initially in, say, the logical 0 state, the output state never contains terms with odd parity, only even parity terms; i.e., the control and target are always either both 0 or both 1 . Thus the correlation between the arms can be quantified by constructing a jointmeasurement fringe visibility in the logical basis, e.g.,

$$
V_{L}=\frac{P_{\text {even }}-P_{\text {odd }}}{P_{\text {even }}+P_{\text {odd }}}=\frac{P_{00}-P_{01}}{P_{00}+P_{01}}=\frac{\left(1+|\varepsilon|^{2}\right)^{-1}}{\left(1+|\varepsilon|^{2}\right)^{-1}}=1,
$$

where $\quad P_{i j}=\operatorname{Tr}(\hat{\rho}|i j\rangle\langle i j|)=\left|\langle i j \mid \psi\rangle_{\text {out }}\right|^{2} \quad$ is the jointmeasurement probability of seeing the state $|i j\rangle$, where $i, j=0$ or 1 . This is a simple indicative measure of a putative CNOT gate: If the logical basis visibility is not unity, then the gate is not an ideal CNOT gate.

To detect entanglement, it is necessary to input and measure superposition states. Analyzing with arbitrary superposition states, $|\theta\rangle_{i}=\cos \theta_{i}|0\rangle_{i}+e^{i \phi_{i}} \sin \theta_{i}|1\rangle_{i}$, where $i$ $=c$ or $t$, the joint-measurement probability becomes ${ }^{7}$

$$
\begin{aligned}
\mathrm{P}_{\theta_{c} \theta_{t}} & =\mid\left\langle\theta_{c}\left|\left\langle\theta_{t} \mid \psi\right\rangle_{\text {out }}\right|^{2}\right. \\
& =\frac{\left|\cos \theta_{c} \cos \theta_{t}+\varepsilon^{*} e^{-i\left(\phi_{c}+\phi_{t}\right)} \sin \theta_{c} \sin \theta_{t}\right|^{2}}{\left(1+|\varepsilon|^{2}\right)} .
\end{aligned}
$$

Now we consider equal-weight superpositions ${ }^{8}$ : Equalweight analysis occurs when $\theta_{c}=\theta_{t}=\pi / 4$, and we define the equal-weight visibility to be

$$
V_{E}\left(\phi_{c}, \phi_{t}\right)=\frac{P_{\phi_{c}, \phi_{t}}^{\pi / 4}-P_{\phi_{c}, \phi_{t}+\pi}^{\pi / 4}}{P_{\phi_{c}, \phi_{t}}^{\pi / 4}+P_{\phi_{c}, \phi_{t}+\pi}^{\pi / 4}}=\frac{2|\varepsilon|}{1+|\varepsilon|^{2}} \cos \left(\phi_{c}+\phi_{t}+\xi\right),
$$

where $\xi=\arg (\varepsilon)$. This fringe visibility is indicative of quantum CNOT-gate operation: If the control state is an equal-weight superposition, $|\varepsilon|=1$, then in the ideal case $\left|V_{E}\right|=1$ (for appropriate $\phi_{c}, \phi_{t}$ ). In the special case where the control and target analyzers have the same phase, $\phi_{c}=\phi_{t}=\phi$, the visibility becomes

$$
V_{E}(\phi)=\frac{2|\varepsilon|}{1+|\varepsilon|^{2}} \cos (2 \phi+\xi) .
$$

A similar measure is the parity, ${ }^{5,9}$ which for two qubits is 


$$
\Pi(\phi)=P_{\phi, \phi}^{\pi / 4}+P_{\phi+\pi, \phi+\pi}^{\pi / 4}-P_{\phi, \phi+\pi}^{\pi / 4}-P_{\phi+\pi, \phi}^{\pi / 4} .
$$

For our example this reduces to $\Pi(\phi)=V_{E}(\phi)$; i.e., for two qubits, the parity is equivalent to the equal-weight visibility. As the phase $\phi$ of the equal-weight analyzers is varied, $V_{E}$ and $\Pi$ oscillate with an amplitude that is twice the magnitude of the extreme off-diagonal element of the density matrix, $|0 \ldots 0\rangle\langle 1 \ldots 1|{ }^{9}$ and a frequency of $2 \phi$. The latter behavior has been used as an indicator of quantum operation, ${ }^{5}$ but care must be exercised when this is used as a measure in the two-qubit case, as separable output states can also give $2 \phi$ fringes [e.g., for $\left|\psi_{i n}\right\rangle=(|0\rangle$ $+|1\rangle)(|0\rangle-|1\rangle) / 2$ the parity of the output is $\Pi(\phi) \propto \cos ^{2} \phi$ $=(1+\cos 2 \phi) / 2$ ]. To unambiguously indicate entanglement, we must combine parity with another measure, such as the logical visibility (see Appendix A for more detail).

\section{Bell-State Fidelity}

An unambiguous entanglement indicator is desirable. One such indicator is the fidelity of any of the two-qubit Bell states, $\left|\phi^{ \pm}\right\rangle=\left(|00\rangle_{ \pm}|11\rangle\right) / \sqrt{2}$ and $\left|\psi^{ \pm}\right\rangle=\left(|01\rangle_{ \pm}|10\rangle\right) / \sqrt{2}$, with the measured state $\hat{\rho}$ :

$$
\begin{aligned}
& F_{\phi^{ \pm}}=\left\langle\phi^{ \pm}|\hat{\rho}| \phi^{ \pm}\right\rangle=\operatorname{Tr}\left(\hat{\rho}\left|\phi^{ \pm}\right\rangle\left\langle\phi^{ \pm}\right|\right), \\
& F_{\psi^{ \pm}}=\left\langle\psi^{ \pm}|\hat{\rho}| \psi^{ \pm}\right\rangle=\operatorname{Tr}\left(\hat{\rho}\left|\psi^{ \pm}\right\rangle\left\langle\psi^{ \pm}\right|\right) .
\end{aligned}
$$

For any separable state, $F_{\phi^{ \pm}, \psi^{ \pm}} \leqslant 1 / 2$ : If $F_{\phi^{ \pm}, \psi^{ \pm}}>1 / 2$, the state is entangled (for instance, see lemma 1 in Ref. 10). It is not necessary to measure the full density matrix $\hat{\rho}$ (which requires 16 joint-measurement probabilities; see Section 3) to determine the Bell fidelities - they can be calculated from just 6 joint-measurement probabilities,

$$
\begin{aligned}
& F_{\phi^{ \pm}}=\left(P_{H H}+P_{V V} \pm P_{D D} \pm P_{A A} \mp P_{R R} \mp P_{L L}\right) / 2, \\
& F_{\psi^{ \pm}}=\left(1 \mp 1-P_{H H}-P_{V V} \pm P_{D D} \pm P_{A A} \pm P_{R R} \pm P_{L L}\right) / 2,
\end{aligned}
$$

where $P_{i i}$ is the joint-measurement probability. We adopt optical-polarization nomenclature as shorthand and define the logical basis as horizontal and vertical, $|H\rangle \equiv|0\rangle \&$ $|V\rangle \equiv|1\rangle$; the equal-weight real superpositions as diagonal and antidiagonal, $|D\rangle=(|0\rangle+|1\rangle) / \sqrt{2}$ and $|A\rangle=(|0\rangle-|1\rangle) / \sqrt{2}$; and the equal-weight complex superpositions as right and left, $|R\rangle=(|0\rangle+i|1\rangle) / \sqrt{2}$ and $|L\rangle=(|0\rangle-i|1\rangle) / \sqrt{2}$. As we shall see in Section 3, even in the presence of experimental uncertainty, the Bell-state fidelity is a reliable and experimentally robust indicator of entanglement. A caveat: Bell fidelities are sensitive only to the state they represent and will miss some entangled states, e.g., the maximally entangled states $(\mathcal{H} \otimes I)\left|\phi^{ \pm}\right\rangle$or $(\mathcal{H} \otimes I)\left|\psi^{ \pm}\right\rangle$, where $\mathcal{H}$ is the Hadamard operation, always result in $F_{\phi^{ \pm}, \psi^{ \pm}} \leqslant 1 / 2$.

It is worth noting here an ongoing ambiguity concerning fidelity: Both Eq. (6) and its square root are referred to in the literature as the fidelity. ${ }^{11}$ Equation (6) has a natural interpretation as the probability of measuring the target stage, e.g., $\left|\phi^{+}\right\rangle$, given the input state, $\rho$ (see Ref. $12)$; the square-root version has no such interpretation. Both definitions are still extant; for example, in Ref. 13 the fidelity of a pair of superconducting qubits with the target state $(|00\rangle+i|11\rangle) / \sqrt{2}$ is given as 0.75 . Squaring this gives the probability of measuring the target state, and the fidelity as defined here is $F=0.56$ - all other things being equal (which is in dispute; see Ref. 14), the measured state is entangled, as $F>1 / 2$.

\section{Entanglement Witness}

More generally, the presence of entanglement can be flagged by an entanglement witness ${ }^{15,16}$-in the usual formulation, an observable $W_{\phi}$ such that $\left\langle W_{\phi}\right\rangle<0$ for some entangled states $|\phi\rangle$ and $\left\langle W_{\phi}\right\rangle \geqslant 0$ for all unentangled states. For every entangled state, a suitable entanglement witness exists; e.g., an experimental study using a witness for $\left|\psi^{-}\right\rangle$was realized in Ref. 17. An optimal entanglement witness for a class of states is one that will detect the entanglement in more states in that class than any other witness.

Since we have a priori knowledge of the entangled states likely to be produced from a CNOT gate, we will concentrate on detecting the entanglement in Werner states, i.e.,

$$
(1-p) / 4 I \otimes I+p|\phi\rangle\langle\phi|
$$

where $p \in[0,1], I \otimes I$ is the maximally mixed state-to model the effect of decoherence-and $|\phi\rangle\langle\phi|$ is the maximally entangled state expected from an ideal CNOT: This state models the effect of a decohering channel. An optimal entanglement witness for this class of states can be simply constructed ${ }^{18}$ by finding the eigenvector $|w\rangle$ corresponding to the minimum eigenvalue of $|\phi\rangle\left\langle\left.\phi\right|^{T_{2}}\right.$, where $T_{2}$ is the partial transpose operation on the second qubit. ${ }^{19}$ The witness is then constructed from $W_{\phi}=|w\rangle\left\langle\left. w\right|^{T_{2}}\right.$. For example, to detect $\left|\phi^{+}\right\rangle$the entanglement witness is

$$
W_{\phi^{+}}=\frac{1}{2}\left[\begin{array}{cccc}
0 & 0 & 0 & -1 \\
0 & 1 & 0 & 0 \\
0 & 0 & 1 & 0 \\
-1 & 0 & 0 & 0
\end{array}\right] .
$$

To measure this witness, we want to decompose it into a sum of local measurements and minimize the number of measurements necessary. It is clear that for two qubits we can decompose the witness operator into tensor products of Pauli matrices-hence only three detector settings are necessary. (In Ref. 18 it was shown that three settings is the minimum that can be achieved.) The following is the decomposition of the witnesses for the four Bell states:

$$
\begin{aligned}
& W_{\phi^{ \pm}}=(I \otimes I \mp X \otimes X \pm Y \otimes Y-Z \otimes Z) / 4, \\
& W_{\psi^{ \pm}}=(I \otimes I \mp X \otimes X \mp Y \otimes Y+Z \otimes Z) / 4,
\end{aligned}
$$

where $X, Y, Z$ are the Pauli spin operators (see Appendix B). These equations show that, as with the Bell-state fidelities, it is not necessary to fully measure the density matrix to measure the witnesses-a set of jointmeasurement probabilities suffices. (This is particularly advantageous for experimental architectures where state tomography is difficult.) For example, by using $\operatorname{Tr}\{\varrho X\}$ $=P_{D}-P_{A}$, where $\varrho$ is a single-qubit density matrix, one such decomposition is

$$
\left\langle W_{\phi^{ \pm}}\right\rangle=\left(P_{H V}+P_{V H} \mp P_{D D} \mp P_{A A} \pm P_{R R} \pm P_{L L}\right) / 2,
$$




$$
\left\langle W_{\psi^{ \pm}}\right\rangle=\left(P_{H H}+P_{V V} \mp P_{D D} \mp P_{A A} \pm P_{R L} \pm P_{L R}\right) / 2 .
$$

Entanglement witnesses can be recast so that $\langle W\rangle\langle b($ $>b)$ for a set of nonseparable states, where $b \in \mathfrak{R}$; and $\langle W\rangle \geqslant b(\leqslant b)$ for all other states (including the separable states). In fact, the Bell-state projectors are such a case of an entanglement witness (see Appendix B), where

$$
\left\langle W_{\phi^{ \pm}, \psi^{ \pm}}\right\rangle=1 / 2-F_{\phi^{ \pm}, \psi^{ \pm}} .
$$

In general, any projective measurement that projects onto an entangled state can be used as an entanglement witness. All entanglement witnesses share the problem highlighted for the Bell-state fidelity-owing to their specificity, they will miss some entangled states.

\section{STATE MEASURES}

Quantitative measures are necessary for measuring, benchmarking, and correcting gate performance. Consider a simple example, where a gate is measured to have logical visibility and parity less than unity. This may occur for a variety of reasons, with the two extremes being that the gate operates nondeterministically as a maximally entangling gate or that the gate operates deterministically, but as a nonmaximally entangling gate. Quantitative measurements distinguish between these alternatives.

\section{A. Quantum-State Tomography}

The measurement of the state of systems of $n$ qubits is a solved problem, comprehensively dealt with in Ref. 20, although open questions remain concerning the optimum measurements and techniques for handling uncertainties. Here we summarize the results relevant to two-qubit gates. As in the last section, we use optical nomenclature for brevity, but this discussion applies equally to all qubits, independent of physical architecture.

The density matrix of a single qubit (or ensemble of identically prepared single qubits) is given by

$$
\hat{\rho}=\frac{1}{2} \sum_{i=0}^{3} \frac{S_{i}}{S_{0}} \hat{\sigma}_{i}
$$

where $\hat{\sigma}_{0}$ is the identity operator and $\hat{\sigma}_{1,2,3}$ are the Pauli spin operators. The single-qubit Stokes parameters are given by, $S_{i}=2 P_{i}-1$, where $P_{i}$ are the measurement probabilities represented by the four projectors $\hat{\mu}_{0}=|H\rangle\langle H|$ $+|V\rangle\left\langle V\left|, \hat{\mu}_{1}=\right| H\right\rangle\left\langle H\left|, \hat{\mu}_{2}=\right| D\right\rangle\langle D|$, and $\hat{\mu}_{3}=|R\rangle\langle R|$. (Note that this set is not unique, and other, more convenient sets may be used depending on architecture. For example, polarization qubits are typically measured using $\hat{\mu}_{0}$ $=|H\rangle\left\langle H\left|, \hat{\mu}_{1}=\right| V\right\rangle\left\langle V\left|, \hat{\mu}_{2}=\right| D\right\rangle\langle D|$, and $\hat{\mu}_{3}=|R\rangle\langle R|$; see Ref. 7.)

Similarly, for two qubits (or identical ensemble of same), the density matrix is given by

$$
\hat{\rho}=\frac{1}{2^{2}} \sum_{i_{1}, i_{2}=0}^{3} \frac{S_{i_{1}, i_{2}}}{S_{0,0}} \hat{\sigma}_{i_{1}} \otimes \hat{\sigma}_{i_{2}},
$$

where the two-qubit Stokes parameters are given by

$$
S_{i_{1}, i_{2}}=\sum_{j_{1}, j_{2}}^{3}\left(\Upsilon^{-1}\right)_{i_{1} j_{1}}\left(\Upsilon^{-1}\right)_{i_{2}, i_{2}} P_{i_{1}, i_{2}}
$$

where

$$
Y^{-1}=\left[\begin{array}{cccc}
1 & 0 & 0 & 0 \\
-1 & 2 & 0 & 0 \\
-1 & 0 & 2 & 0 \\
-1 & 0 & 0 & 2
\end{array}\right], \quad \hat{\mu}_{i} \otimes \hat{\mu}_{j}(i, j=0,1,2,3)
$$

are projectors representing the 16 joint-measurement probabilities, $P_{i_{1}, i_{2}}$.

\section{B. Physical Quantum-State Tomography}

Equations (13) and (14) are not applicable in the presence of experimental uncertainties-such as those introduced by count statistics or analyzer uncertainties. If used in such a case, they can lead to nonphysical density matrices that violate properties such as positivity. This can be avoided by using maximum-likelihood estimation techniques based on the above probability measurements. ${ }^{20,21}$ These techniques find the closest physical state that is consistent with the observed probabilities by minimizing some objective function, e.g., least squares. (A more detailed discussion can be found in the next section, concerning physical process tomography.) The method in Ref. 20 is not guaranteed to find the global minimum. In recent developments, it has been shown that the problem can be recast as a convex optimization, ensuring that the global minimum is found efficiently. ${ }^{21}$ This remains an active area of research, e.g., with a recent proposal to replace maximum-likelihood with Bayesian-state estimation. $^{22}$

\section{Entropy}

Having measured a density matrix, it is instructive to consider what physical information can be extracted from it. The most obvious attribute is the degree of order, or entropy. For a pure state, the degree of order is maximal and the entropy is zero. Entropy measures used to date for a state $\rho$ include the von Neumann entropy, $S$ $=-\operatorname{Tr}\left[\rho \log _{2} \rho\right]$, and linear entropy, $S_{L}=4\left(1-\operatorname{Tr}\left[\rho^{2}\right]\right) / 3$. In the latter case, the entropy is scaled between $S_{L}=0$ (for a pure state) and $S_{L}=1$ (for a fully mixed state). ${ }^{23}$ For perfect CNOT operation and pure input states, all output states remain pure and the measured entropies will be zero.

Single-qubit density matrices can be uniquely decomposed into a maximally mixed component $\left(S_{L}=1\right)$ and a completely pure component $\left(S_{L}=0\right)$; e.g., polarized light can be described as a specific combination of unpolarized and purely polarized light. It is tempting to decompose two-qubit density matrices in a similar fashion; however, in this case no unique decomposition exists and infinitely many possible combinations are possible. It is not possible to uniquely characterize the action of any two-qubit gate in this manner.

\section{Tangle}

As we have seen, the other physically significant attribute of two-qubit systems is the degree of entanglement. There 


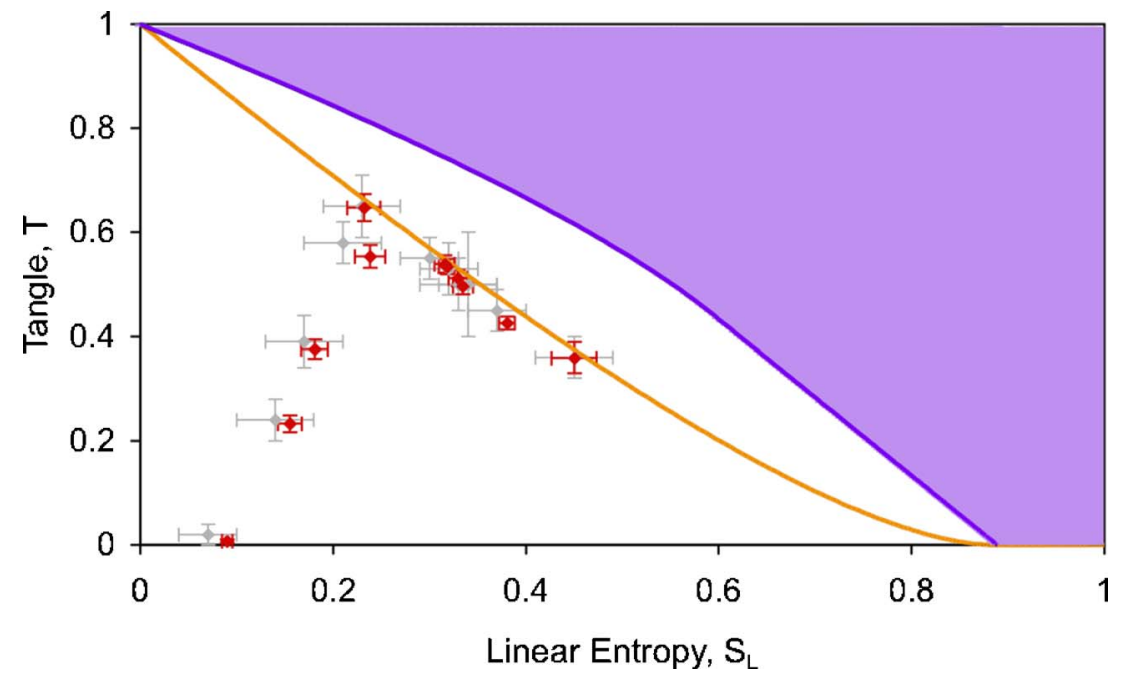

Fig. 1. (Color online) Tangle-entropy plane. Measurements were made of 11 different two-qubit states (in this case, polarizationentangled photons). For each state, density matrices were obtained using the tomographic methods of Ref. 20 (gray points, error bars calculated analytically) and Ref. 21 [black points (red online), error bars calculated via a Monte Carlo technique ${ }^{29}$ ]. The upper curved line indicates maximally entangled mixed states ${ }^{27}$ (values above this bound are unphysical); the lower curve indicates Werner states [Eq. (8)].

is an active literature on measures for quantifying entanglement: Without loss of generality, we consider here the tangle, which is a measure that detects any degree of entanglement, for all two-qubit states, be they pure or mixed. ${ }^{24,25}$ It is quantitative, varying between $T=0$ for a unentangled state to $T=1$ for a maximally entangled state. For the special case of pure states-unlikely in practice- the tangle is simply given by

$$
T=V_{E}(n \pi / 2)^{2}=\Pi(n \pi / 2)^{2},
$$

for $n \in \mathcal{N}$. More generally, for a density matrix in the logical basis representation, $\rho$, the spin-flipped matrix is defined to be $\widetilde{\rho}=(Y \otimes Y) \hat{\rho}^{*}(Y \otimes Y),{ }^{25}$ where $\hat{\rho}^{*}$ is the complex conjugate of $\hat{\rho}$.

Here $\left\{\lambda_{1}, \lambda_{2}, \lambda_{3}, \lambda_{4}\right\}$ are the eigenvalues of $\hat{\rho} \tilde{\rho}$ in decreasing order, and the tangle is

$$
T=\left(\max \left\{\lambda_{1}-\lambda_{2}-\lambda_{3}-\lambda_{4}, 0\right\}\right)^{2} .
$$

Amongst other properties, the tangle is a conserved measure of the amount of entanglement that can be shared between multiple qubits, and the tangle is well defined for two-qubit mixed states.

The salient attributes of a two-qubit state can be characterized by its location on the tangle-entropy plane. ${ }^{23,26}$ The output states of an ideal CNOT gate lie on the ordinate axis; nonideal operation introduces decoherence, and the measured output states move from the axis into the plane. There is a limit to the amount of entanglement possible for a given amount of decoherence. States that have the maximum amount are known as maximally entangled mixed states (MEMS) and form a boundary on the tangleentropy plane. ${ }^{27,28}$

Figure 1 shows a range of two-qubit states of varying entropy and tangle (in this case polarization-entangled photons obtained in our laboratory, using an experimental system essentially identical to that described in Refs. 7 and 23). Each data point comes from an experimentally derived density matrix, constructed from 16 measurements as described above (also see Ref. 29). Reference 20 has modeled the effect of experimental uncertainties on a number of quantities derived from the density matrix, including the entropy and tangle, making them suitable quantities to use in characterizing experimental systems.

Figure 2 lists the Bell-state fidelities for these matrices-both those obtained with six measurements [Eq. (7)] and those obtained via the density matrix. ${ }^{20,21}$ Despite the presence of experimental uncertainties, the fidelity reliably witnesses the entanglement in every case. The 6-measurement fidelity has less uncertainty than the 16-measurement one-this may be because in the latter case a more complicated algorithm is used to estimate the error $^{20}$ or extra uncertainty is introduced by measuring more parameters than required to find the fidelity (16 versus 6) or some combination of the two.

Figure 2 underscores the point made earlier that that the Bell-state fidelities-indeed, entanglement witnesses of any kind-are not metrics for entanglement; i.e., they do not provide a measure of how entangled a state is, they merely provide an indicator that the state is entangled.

\section{E. Bell's Inequality}

Historically, the presence of entanglement in an experimental system was indicated by the violation of Bell's inequality, $B .^{30,31}$ Such a violation is striking, as it rules out a wide class of possible "classical" models. ${ }^{32}$ For states that can be described by classical local hidden-variable models, $B \leqslant 2$, whereas for a certain range of quantum states, including maximally entangled states, $B>2$; for maximally entangled states, $B=2 \sqrt{2}$. In general, Bell's inequality is an indicator of entanglement, not a measure, as it is a function of both entanglement and entropy. ${ }^{26}$ This limits its utility for gate characterization, as it does not distinguish between coherent and incoherent phenomena. Only for the special case of pure states is Bell's inequality a measure, related to the tangle by Ref. $26, B$ $=2 \sqrt{1+T^{2}}$. 


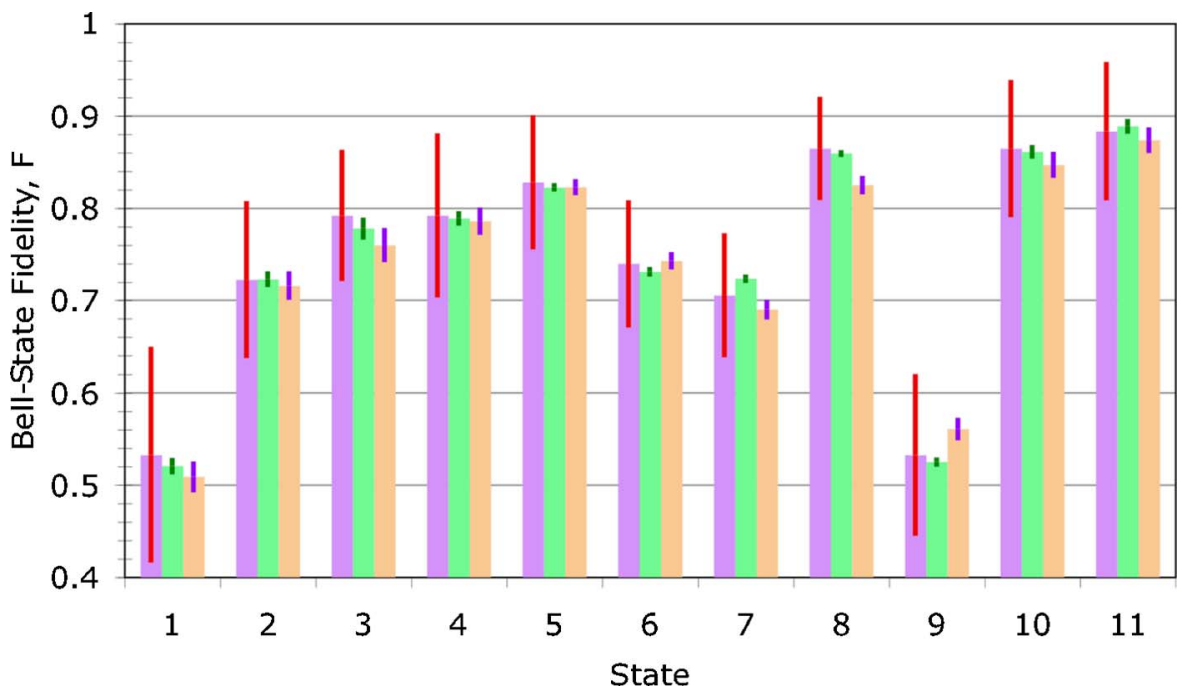

Fig. 2. (Color online) Bell-state fidelities for Fig. 1, where $F>1 / 2$ indicates entanglement. For each state, the left and center bars were calculated using density matrices obtained via the tomographic methods of Refs. 20 and 21, respectively; right bars were calculated from the entanglement witness obtained by six measurements [Eq. (7)]. Uncertainties for each fidelity are shown by thin vertical lines. Note that the Bell-state fidelity is an entanglement indicator, not a measure; e.g., states 1 and 9 have similar fidelities but very different tangles, $\sim 0.01$ and $\sim 0.54$, respectively (data in Table 4 in Appendix B).

\section{PROCESS MEASURES}

\section{A. Quantum Process Tomography}

Measuring a state is not the same as measuring a gate. Just as measuring a set of output probabilities allows reconstruction of the gate output state, measuring a set of gate output states enables reconstruction of the gate process.

Any quantum process or operation $\mathcal{E}$ can be written in the operator-sum representation for an arbitrary input state $\rho$ :

$$
\mathcal{E}(\rho)=\sum_{k} E_{k} \rho E_{k}^{\dagger}
$$

where $E_{k}$ are known as operation elements or Kraus operators and have the condition that $\Sigma_{j} E_{j}^{\dagger} E_{j} \leqslant I$, with equality for trace preserving maps. The set of operation elements $\left\{E_{k}\right\}$ completely describes the effect of the operation, including unitary evolution, measurement, and decoherence. ${ }^{33}$ With process tomography, we want to experimentally determine some $E_{k}$ matrices that represent the process.

There is a spectrum of approaches to process tomography. (1) Single-input ancilla-assisted process tomography, ${ }^{34-36}$ where state tomography is carried out on the output of the process $(\mathcal{E} \otimes I)(\sigma)$ on a single input state $\sigma$ of the original system combined with an ancilla, or auxilliary, system. (2) Standard quantum process tomography (SQPT; see Refs. 37-42) in which state tomography is carried out on each result from a set of input states $\left\{\rho_{i}\right\}$. (3) Single-measurement ancilla-assisted process tomography, ${ }^{43,44}$ where a single joint measurement of the original system combined with an ancilla system is performed on a set of input states $\left\{\rho_{j}\right\}$, where $\left\{\rho_{j}\right\}>\left\{\rho_{i}\right\}$.

We present only SQPT here. All variations achieve the same end, but for two-qubit gates the advantage of SQPT is that both the input states and measurements are straightforward to achieve. We first present the general formulation of the technique and follow this with a much simpler formulation for two qubits where we have chosen a particular basis. Our discussion largely follows references 1 and 37. SQPT is performed in the following steps:

1. Choose a fixed basis of linearly independent input states $\left\{\widetilde{\rho}_{i}\right\}$ and experimentally determine the output density operators $\mathcal{E}\left(\widetilde{\rho}_{i}\right)$ for each using state tomography. These can be expressed in the basis as

$$
\mathcal{E}\left(\widetilde{\rho}_{i}\right)=\sum_{j} \lambda_{i j} \widetilde{\rho}_{j}
$$

2. Also choose a fixed basis $\left\{\widetilde{E}_{j}\right\}$ for the operators on the state space. Express the operation elements in Eq. (18) in this basis, $E_{i}=\Sigma_{m} a_{i m} \widetilde{E}_{m}$, hence

$$
\mathcal{E}(\rho)=\sum_{m n} \chi_{m n} \widetilde{E}_{m} \rho \widetilde{E}_{n}^{\dagger},
$$

where $\chi_{m n}$ is a positive Hermitian matrix, $\chi_{m n} \equiv \Sigma_{i} a_{i m} a_{i n}^{*}$, that completely describes the process in the chosen basis.

3. Now, writing $\widetilde{E}_{m} \widetilde{\rho}_{i} \widetilde{E}_{n}^{\dagger}=\Sigma_{j} \beta_{i j}^{m n} \widetilde{\rho}_{j}$, express the process for the input states as

$$
\mathcal{E}\left(\widetilde{\rho}_{i}\right)=\sum_{j m n} \chi_{m n} \beta_{i j}^{m n} \tilde{\rho}_{j}
$$

Comparing Eqs. (19) and (21) and using the fact that the $\tilde{\rho}_{i}$ are a basis yields $\lambda_{i j}=\Sigma_{m n} \beta_{i j}^{m n} \chi_{m n}$. Combining the indices $i j$ and $m n$ (e.g., $\{11,21,31,41,12, \ldots, 44\}$ ), gives the matrix equation $\vec{\lambda}=\beta \vec{\chi}$. Hence, by inverting $\beta, \vec{\chi}$ can be found in terms of the experimentally determined $\vec{\lambda}$.

4. As mentioned previously, $\chi$ is positive: Using the spectral theorem, decompose it into a product of some unitary operator $U$ and a positive diagonal matrix $D, \chi$ $=U D U^{\dagger}$. This constructs the operation elements,

$$
E_{k}=\sqrt{D_{k k}} \sum_{m} U_{m k} \widetilde{E}_{m}
$$


For particular cases we can choose the basis so that the above calculations are easily performed. Consider a twoqubit process or gate. If the basis for the input states is given by $\rho_{m n}^{(j k)} \equiv \delta_{j m} \delta_{k n}$, where $\rho^{(j k)}$ is a matrix with a 1 on the $j$ th row and $k$ th column and zeros elsewhere; and we choose as our operator basis $\widetilde{A} \otimes \widetilde{B}$ where $\widetilde{A}, \widetilde{B}$ $\in\{I, X, Y, Z\}$, then the $16 \times 16 \chi$-matrix is easily constructed in block form:

$$
\chi=K^{T}\left[\begin{array}{ccc}
\mathcal{E}\left(\rho^{(11)}\right) & \mathcal{E}\left(\rho^{(12)}\right) & \cdots \\
\mathcal{E}\left(\rho^{(21)}\right) & \mathcal{E}\left(\rho^{(22)}\right) & \cdots \\
\vdots & \vdots & \ddots
\end{array}\right] K,
$$

where $K$ is a particular matrix constructed from $K=P \Lambda$, with $P=I \otimes\left[\rho^{(11)}+\rho^{(23)}+\rho^{(32)}+\rho^{(44)}\right] \otimes I$ and $\Lambda=(Z \otimes I+X$ $\otimes X) \otimes(Z \otimes I+X \otimes X) / 4$.

The $\rho^{(j k)}$ basis operators for the initial states do not necessarily themselves represent physical states. This is not a problem, as we can use the linearity of the operation $\mathcal{E}(\rho)$ to construct them from suitable combinations of other operators. In Appendix $\mathrm{C}$ we relate $\rho^{(j k)}$ to the $\{|H\rangle$ $\times\langle H|| V\rangle,\langle V|| D\rangle,\langle D|| R\rangle,\langle R|\}$ operators. In Appendix D we give a detailed example of process tomography for a twoqubit gate.

\section{B. Physical Quantum Process Tomography}

As was the case for state tomography, in practice the process matrix reconstructed by the above technique will not represent a physical process. Unavoidable experimental uncertainties in the measurement statistics will lead to sets of probabilities that are inconsistent with a true physical process, and the reconstructed Kraus operators will not fullfil the property that $\Sigma_{j}^{\dagger} E_{j} \leqslant I$.

To properly reconstruct the Kraus operators in the presence of noise, one can use maximum-likelihood estimation. The procedure is to parametrize a general process $\mathcal{E}_{\vec{x}}$ by a set of parameters $\vec{x}$ in such a way that it always represents a physical process. Assuming Poissonian uncertainties in the Gaussian limit, the probability of obtaining a particular set of outcomes, $p_{a b}$, given a set input states, $\left\{\rho_{a}\right\}$, and measurements, $\left\{M_{b}\right\}$, is given by

$$
P\left(\left\{p_{a b}\right\} \mid \mathcal{E}_{\vec{x}}\right) \propto \prod_{a b} \exp \left[-\frac{\left(p_{a b}-\operatorname{Tr}\left\{M_{b} \mathcal{E}_{\vec{x}}\left(\rho_{a}\right)\right\}\right)^{2}}{2 \operatorname{Tr}\left\{M_{b} \mathcal{E}_{\vec{x}}\left(\rho_{a}\right)\right\}}\right]
$$

This probability is known as the likelihood function: Maximum-likelihood estimation involves maximizing this probability over all parameters $\vec{x}$, which are related to the $\chi$-matrix of the previous section. This can be simplified by taking the negative logarithm of the likelihood, leading to the following minimization ${ }^{42}$ :

$$
\min _{\vec{x}} \sum_{a b} \frac{\left(p_{a b}-\operatorname{Tr}\left\{M_{b} \mathcal{E}_{\vec{x}}\left(\rho_{a}\right)\right\}\right)^{2}}{2 \operatorname{Tr}\left\{M_{b} \mathcal{E}_{\vec{x}}\left(\rho_{a}\right)\right\}} .
$$

Again, as with the state tomography case, this problem can be reduced to a convex-optimization problem, ${ }^{21}$ with the concomitant benefit of efficient calculation of the glo- bal minimum. This technique was recently used to measure physical $\chi$-matrices for an experimental CNOT gate. ${ }^{45}$

\section{Metrics}

Having obtained the operator elements, $\chi$, we must determine how close the process is to the ideal gate that we are trying to implement? Further, what determines whether a given measure is useful? Six physically motivated requirements have been identified for such measures. ${ }^{11}$ They should (i) be a metric (behave like a ruler); (ii) be easy to calculate; (iii) be easy to measure; (iv) have a nice physical interpretation; (v) be stable (unrelated ancillary quantum systems should not affect the value); and (vi) obey chaining (for a process composed of many smaller steps, the total error between that process and some ideal process will be less than the sum of the errors in the individual steps and their ideal steps).

Four metrics have been identified that satisfy the above requirements. ${ }^{11}$ For a process, $\mathcal{E}$, and a target unitary, $U$, the process fidelity and distance, $F_{p}$ and $D_{p}$, respectively, and the stabilized fidelity and distance, $F_{s}$ and $D_{s}$, respectively, are

(i) $F_{p}(\mathcal{E}, U) \equiv F\left(\rho_{\mathcal{E}}, \rho_{U}\right)$,

(ii) $D_{p}(\mathcal{E}, U) \equiv D\left(\rho_{\mathcal{E}}, \rho_{U}\right)$,

(iii) $D_{s}(\mathcal{E}, U) \equiv \max _{\psi} D[(I \otimes \mathcal{E})(\psi),(I \otimes U)(\psi)]$,

(iv) $F_{s}(\mathcal{E}, U) \equiv \max _{\psi} F[(I \otimes \mathcal{E})(\psi),(I \otimes U)(\psi)]$,

where $F$ and $D$ are the state fidelity (Section 2) and trace distance, $D(\rho, \sigma)=\operatorname{Tr}|\rho-\sigma| / 2$ (see Ref. 1) and $\psi=|\psi\rangle\langle\psi|$, respectively. Note that $F_{p}$ and $D_{p}$ exploit an isomorphism between quantum processes and unnormalized states, ${ }^{46,47}$ where we can construct a positive state, $\rho_{\mathcal{E}}$, from the action of the process on part of the maximally entangled state $\left|\Psi^{+}\right\rangle=\Sigma_{j}|j\rangle|j\rangle / \sqrt{d}$ of a $d$-dimensional system, $\rho_{\mathcal{E}}=(I$ $\otimes \mathcal{E})\left|\Psi^{+}\right\rangle\left\langle\Psi^{+}\right|$

An operational interpretation for $F_{p}$ and $D_{p}$ is that they bound on the average probability of error, $\bar{P}_{e}$, experienced during quantum computation of a function, i.e.,

$$
\begin{aligned}
\bar{P}_{e} & \leqslant D_{p}(\mathcal{E}, U) \\
& \leqslant 1-F_{p}(\mathcal{E}, U) .
\end{aligned}
$$

Here $D_{s}$ and $F_{s}$ have an even stronger physical interpretation. Both metrics can be interpreted as a bound on the worst-case error probability $P_{e}$ for a function computation:

$$
\begin{aligned}
P_{e} & \leqslant D_{s}(\mathcal{E}, U) \\
& \leqslant 1-F_{s}(\mathcal{E}, U),
\end{aligned}
$$

where $F_{p}$ can be simply related to the average gate fidelity, $\bar{F}$, which is the fidelity between the target process, $\mathcal{F}(\psi)$ and the measured process, $\mathcal{E}(\psi)$, averaged over all possible gate inputs, $\psi{ }^{1}$ 


$$
\bar{F}(\mathcal{E}, \mathcal{F}) \equiv \int \mathrm{d} \psi F(\mathcal{E}(\psi), \mathcal{F}(\psi))
$$

When $\mathcal{F}$ is a unitary operation, $U, \bar{F}$, and $F_{p}$ are related by the following equation ${ }^{48,49}$ :

$$
\bar{F}(\mathcal{E}, U)=\frac{d F_{p}(\mathcal{E}, U)+1}{d+1}
$$

where $d$ is the dimension of the quantum system. Experimentally, $\bar{F}$ is as easy to measure as $F_{p}$.

Indeed $F_{p}$ can be determined without full process tomography ${ }^{11,42}$ requiring only $d^{2}$ to $2 d^{2}$ measurements for a $d$-dimensional Hilbert space, i.e., 16-32 measurements for a two-qubit gate. ${ }^{11}$ Not only is this much simpler to obtain experimentally, it allows straightforward determination of experimental uncertainties. This can be seen by representing the $\chi$-matrix in a basis where the first element represents the target gate, e.g., for $\left\{\text { CNOT } \sigma_{j} \otimes \sigma_{k}\right\}_{j k}$ the first element is CNOT $I \otimes I$. Since the height of this element is $F_{p},{ }^{42}$ only a small set of measurements is needed to determine it. Bounds on $F_{p}$ can be obtained with even fewer, $2 d$, measurements. ${ }^{50,51}$

In contrast, there is no known elementary formula for

Table 3. Table of Two-Qubit Gate Measures ${ }^{a}$

\begin{tabular}{lcccc}
\hline Measure Type & $\begin{array}{c}\text { No. of } \\
\text { measure- } \\
\text { ments Name }\end{array}$ & Symbol & $\begin{array}{c}\text { Lower } \\
\text { Bound }\end{array}$ & $\begin{array}{c}\text { Ideal } \\
\text { Value }\end{array}$ \\
\hline Indicative measures & & & & \\
$\quad$ Inquisition & 4 & $\mathcal{I}$ & 0 & 1 \\
Logical visibility & 2 & $V_{L}$ & 0 & \pm 1 \\
Equal-weight visibility & 2 & $V_{E}\left(n \frac{\pi}{2}\right)$ & 0 & \pm 1 \\
& & & & \\
Parity & 4 & $\Pi\left(n \frac{\pi}{2}\right)$ & 0 & \pm 1 \\
& & & & \\
Bell-state fidelity & 6 & $F_{\phi^{ \pm}, \psi^{ \pm}}$ & $\frac{1}{2}$ & 1 \\
Entanglement witness & 6 & $\left\langle W_{\phi^{ \pm}, \psi^{ \pm}}\right\rangle$ & 0 & $-\frac{1}{2}$ \\
State measures & & & & \\
Density matrix & 16 & $\hat{\rho}$ & & \\
Linear entropy & 16 & $S_{L}$ & 1 & 0 \\
Tangle & 16 & $T$ & 0 & 1 \\
.. for pure states & & $V_{E}^{2}, \Pi^{2}$ & & \\
Bell's inequality & 16 & $B$ & 2 & $2 \sqrt{2}$ \\
$\quad$.. for pure states $=$ & & $2 \sqrt{1+T^{2}}$ & & \\
Process measures & & & & \\
Chi matrix & 256 & $\chi$ & & \\
Process fidelity & $16-32$ & $F_{p}$ & 0 & 1 \\
Process distance & 256 & $D_{P}$ & 1 & 0 \\
Stabilized fidelity & 256 & $F_{s}$ & 0 & 1 \\
Stabilized distance & 256 & $D_{s}$ & 1 & 0 \\
Average gate fidelity & $16-32$ & $\bar{F}$ & 0 & 1 \\
\hline
\end{tabular}

${ }^{a}$ Table gives number of measurements required for each measure and the lower and ideal bounds of the measure, if any. Indicative measures indicate gate logical operation or the presence of entanglement in the gate output. State measures provide quantitative measurements of the gate output states. Process measures provide quantitative measurements of the gate itself.
$D_{s}$ or $F_{s}$, but calculating these distances is equivalent to convex-optimization problems, which can be efficiently solved numerically. For all these metrics, there are open questions in applying them to experimentally characterizing gates. Most notably, how do uncertainties in state preparation and tomography finally manifest themselves in the measure?

\section{CONCLUSION}

We have discussed a range of architecture-independent measurements for two-qubit gates that vary along a sliding scale of complexity. In this paper we have illustrated these for the example of a controlled-NOT gate, summarized in Table 3: Similar arguments can be applied to derive appropriate measures for any two-qubit entangling gate. Simpler measures, such as indicative and state measures, are suitable for early diagnosis and developmental stages of a quantum-logic gate. For definitive statements regarding gate operation, and to allow predictions of scaling behavior, quantitative process measures must be used since only they can provide full functional gate descriptions and bounds to error behaviors.

\section{APPENDIX A: PARITY}

The parity, $\Pi$, should not be used on its own to indicate quantum gate operation: It should be combined with another indicator (such as the logical visibility, $V_{L}$ ) and applied over a range of input states.

Consider a semiclassical, CNOT-like gate, which measures the control qubit, applying a bit flip, $X$, if the results is 1 . It is described by the completely positive map,

$$
\mathcal{E}(\hat{\rho})=\left(K_{0} \otimes I\right) \hat{\rho}\left(K_{0} \otimes I\right)+\left(K_{1} \otimes X\right) \hat{\rho}\left(K_{1} \otimes X\right),
$$

where $K_{0}=|0\rangle\langle 0|$ and $K_{1}=|1\rangle\langle 1|, \hat{\rho}$ is the density matrix of the input state, and the one-qubit identity operator, $I$, and the Pauli spin operators, $X, Y, Z$ are given by $I \equiv \hat{\sigma}_{0}$ $=\left[\begin{array}{ll}1 & 0 \\ 0 & 1\end{array}\right], X \equiv \hat{\sigma}_{1}=\left[\begin{array}{cc}0 & 1 \\ 1 & 0\end{array}\right], Y \equiv \hat{\sigma}_{2}=\left[\begin{array}{cc}0 & -i \\ i & 0\end{array}\right], Z \equiv \hat{\sigma}_{3}=\left[\begin{array}{cc}1 & 0 \\ 0 & -1\end{array}\right]$. Gate (A1) reproduces the action of a CNOT gate for all computational-basis inputs, $V_{L}=1$, and all targetsuperposition inputs. However, it fails the parity indicator for control-superposition inputs, yielding $\Pi_{\max }=0$, since the gate destroys the superposition in the control qubit.

This can be remedied by inverting the gate orientation by applying Hadamard gates $(\mathcal{H})$ before and after

$$
\begin{aligned}
\mathcal{E}(\hat{\rho})= & \left(I \otimes \mathcal{H} K_{0} \mathcal{H}\right) \hat{\rho}\left(I \otimes \mathcal{H} K_{0} \mathcal{H}\right)+\left(Z \otimes \mathcal{H} K_{1} \mathcal{H}\right) \hat{\rho}(Z \\
& \left.\otimes \mathcal{H} K_{1} \mathcal{H}\right) .
\end{aligned}
$$

This gate is semiclassical, yet it has the same maximum parity, $\Pi_{\max }=1$, and the same number of parity fringes as an ideal CNOT gate: Parity alone cannot be used as an in- 
Table 4. Values for Figs. 1 and $2^{a}$

\begin{tabular}{|c|c|c|c|c|c|c|c|}
\hline \multirow[b]{2}{*}{ State } & \multicolumn{3}{|c|}{ Ref. 20} & \multicolumn{3}{|c|}{ Ref. 21} & \multirow{2}{*}{$\begin{array}{c}\text { Entanglement Witness } \\
\text { Bell-state } \\
\text { Fidelity, } F_{\phi^{ \pm}}\end{array}$} \\
\hline & $\begin{array}{c}\text { Entropy } \\
S_{L}\end{array}$ & $\begin{array}{c}\text { Tangle } \\
T\end{array}$ & $\begin{array}{c}\text { Bell-State } \\
\text { Fidelity, } F_{\phi^{ \pm}}\end{array}$ & $\begin{array}{c}\text { Entropy } \\
S_{L}\end{array}$ & $\begin{array}{c}\text { Tangle } \\
\quad T \\
\end{array}$ & $\begin{array}{c}\text { Bell-State } \\
\text { Fidelity, } F_{\phi^{ \pm}}\end{array}$ & \\
\hline 1 & $0.07(3)$ & $0.02(2)$ & $0.53(12)_{\phi^{+}}$ & $0.090(5)$ & $0.007(3)$ & $0.521(9)_{\phi^{+}}$ & $0.509(17)_{\phi^{+}}$ \\
\hline 2 & $0.14(4)$ & $0.24(4)$ & $0.72(9)_{\phi^{+}}$ & $0.155(12)$ & $0.233(16)$ & $0.723(8)_{\phi^{+}}$ & $0.716(15)_{\phi^{+}}$ \\
\hline 3 & $0.45(4)$ & $0.36(4)$ & $0.79(7)_{\phi^{+}}$ & $0.450(24)$ & $0.359(29)$ & $0.778(12)_{\phi^{+}}$ & $0.760(18)_{\phi^{+}}$ \\
\hline 4 & $0.17(4)$ & $0.39(5)$ & $0.79(9)_{\phi^{+}}$ & $0.181(14)$ & $0.376(19)$ & $0.789(8)_{\phi^{+}}$ & $0.786(15)_{\phi^{+}}$ \\
\hline 5 & $0.37(3)$ & $0.45(4)$ & $0.83(7)_{\phi^{+}}$ & $0.380(8)$ & $0.426(12)$ & $0.823(5)_{\phi^{+}}$ & $0.823(9)_{\phi^{+}}$ \\
\hline 6 & $0.33(4)$ & $0.50(5)$ & $0.74(7)_{\phi^{+}}$ & $0.355(11)$ & $0.496(16)$ & $0.731(5)_{\phi^{+}}$ & $0.743(9)_{\phi^{+}}$ \\
\hline 7 & $0.34(3)$ & $0.50(10)$ & $0.71(7)_{\phi^{-}}$ & $0.329(10)$ & $0.512(16)$ & $0.724(4)_{\phi^{-}}$ & $0.690(11)_{\phi^{-}}$ \\
\hline 8 & $0.32(3)$ & $0.53(5)$ & $0.86(6)_{\phi^{-}}$ & $0.318(8)$ & $0.534(15)$ & $0.859(4)_{\phi^{-}}$ & $0.825(10)_{\phi^{-}}$ \\
\hline 9 & $0.30(3)$ & $0.55(4)$ & $0.53(9)_{\phi^{+}}$ & $0.316(11)$ & $0.539(17)$ & $0.525(5)_{\phi^{+}}$ & $0.561(12)_{\phi^{+}}$ \\
\hline 10 & $0.21(4)$ & $0.58(4)$ & $0.86(7)_{\phi^{+}}$ & $0.238(16)$ & $0.554(22)$ & $0.861(7)_{\phi^{+}}$ & $0.847(14)_{\phi^{+}}$ \\
\hline 11 & $0.23(4)$ & $0.65(6)$ & $0.88(8)_{\phi^{+}}$ & $0.232(17)$ & $0.647(26)$ & $0.889(8)_{\phi^{+}}$ & $0.874(14)_{\phi^{+}}$ \\
\hline
\end{tabular}

${ }^{a}$ Calculated using the techniques of Refs. 20 and 21 and the direct measurements of the entanglement witness. Bracketed figures are uncertainty in the last significant figures.

dicator of quantum operation. Measuring in the computational basis yields $V_{L}=0$, distinguishing this gate from a CNOT gate that would yield $V_{L}=1$. Measurement of both the computational basis and the parity are required. ${ }^{5}$

\section{APPENDIX B: ENTANGLEMENT WITNESSES AND BELL-STATE FIDELITY}

Interestingly, the witnesses of Eq. (11) can be written as

$$
\begin{aligned}
& W_{\phi^{ \pm}}=\left(\left|\psi^{ \pm}\right\rangle\left\langle\psi^{ \pm}\right|\right)^{T_{2}}=1 / 2 I \otimes I-\left|\phi^{ \pm}\right\rangle\left\langle\phi^{ \pm}\right|, \\
& W_{\psi^{ \pm}}=\left(\left|\phi^{ \pm}\right\rangle\left\langle\phi^{ \pm}\right|\right)^{T_{2}}=1 / 2 I \otimes I-\left|\psi^{ \pm}\right\rangle\left\langle\psi^{ \pm}\right| .
\end{aligned}
$$

If we note that the condition for the detection of an entangled state is $\left\langle W_{\psi^{ \pm}, \phi^{ \pm}}\right\rangle=\operatorname{Tr}\left(W_{\psi^{ \pm}, \phi^{ \pm}} \hat{\rho}\right)<0$, then we see

$$
\begin{aligned}
\operatorname{Tr}\left(W_{\psi, \phi} \hat{\rho}\right) & =1 / 2-\operatorname{Tr}(\hat{\rho}|\psi, \phi\rangle\langle\psi, \phi|) \\
& =1 / 2-F_{\psi^{ \pm}, \phi^{ \pm}} .
\end{aligned}
$$

So if $\left\langle W_{\psi, \phi}\right\rangle<0$, we see that this is equivalent to $F_{\psi, \phi}$ $>1 / 2$, which is simply the Bell-state fidelity entanglement indicator. Generally, we can derive an optimal class of entanglement witnesses for any two-qubit maximally entangled state in a similar fashion. We note that all of the maximally entangled states on two qubits can be generated from one of the Bell states and the application of a single local unitary gate, for instance, $U \otimes I\left|\phi^{+}\right\rangle$. Then from Ref. 18 we know that the optimal witness for the state $(1-p) I \otimes I+p\left|\phi^{+}\right\rangle\left\langle\phi^{+}\right|$is $\left(\left|\psi^{-}\right\rangle\left\langle\psi^{-}\right|\right)^{T_{2}}$. This is found as $\left|\psi^{-}\right\rangle$, which is the eigenvector corresponding to the minimum eigenvalue of the matrix $\left(\left|\phi^{+}\right\rangle\left\langle\phi^{+}\right|\right)^{T_{2}}$. That is,

$$
\left(\left|\phi^{+}\right\rangle\left\langle\phi^{+}\right|\right)^{T_{2}}\left|\psi^{-}\right\rangle=w\left|\psi^{-}\right\rangle \text {. }
$$

If we multiply from the left by $U \otimes I$, we get

$$
\left.U \otimes I\left(\left|\phi^{+}\right\rangle\left\langle\phi^{+}\right|\right)^{T_{2}}\left|\psi^{-}\right\rangle=w U \otimes I \psi^{-}\right\rangle .
$$

Then if we insert an identity and note that $U \otimes I$ commutes with the partial transpose operation, we find

$$
\left(U \otimes I\left|\phi^{+}\right\rangle\left\langle\phi^{+}\right| U^{\dagger} \otimes I\right)^{T_{2}} U \otimes I\left|\psi^{-}\right\rangle=w U \otimes I\left|\psi^{-}\right\rangle ;
$$

hence the optimal witness for an arbitrary Werner state, $(1-p) I \otimes I+p U \otimes I\left|\phi^{+}\right\rangle\left\langle\phi^{+}\right| U^{\dagger} \otimes I, \quad$ is $\left(U \otimes I\left|\psi^{-}\right\rangle\left\langle\psi^{-}\right| U^{\dagger} \otimes I\right)^{T_{2}}$. Then Eq. (B1) tells us that this witness can be written as $1 / 2 I \otimes I-U \otimes I\left|\phi^{+}\right\rangle\left\langle\phi^{+}\right| U^{\dagger} \otimes I$.

Hence we see given some Werner state of the form $p I$ $\otimes I+(1-p)|\theta\rangle\langle\theta|$, the optimal witness for the state as defined in Ref. 18, is simply $W=1 / 2 I \otimes I-|\theta\rangle\langle\theta|$, where $|\theta\rangle$ is some maximally entangled state. Thus the value of an optimal Werner witness measurement $\langle W\rangle$ is always linearly related to the square of the fidelity between a maximally entangled state and the state being analyzed. This, allows us to interpret this witness as the witness for which the fidelity between the output state and some maximally entangled state is greater than $1 / 2$.

In general, any projective measurement that projects onto an entangled state can be used as an entanglement witness in a similar fashion. It is relatively easy to prove that given some entangled state $|\psi\rangle$, with maximum Schmidt coefficient, $\lambda_{\text {max }}$, then $\operatorname{Tr}\left(\hat{\rho}_{\text {sep }}|\psi\rangle\langle\psi|\right) \leqslant \lambda_{\text {max }}^{2}$ for all separable states, $\hat{\rho}_{\text {sep }}$. Thus an entanglement witness $W$ $=\lambda_{\max }^{2} I \otimes I-|\psi\rangle\langle\psi|$ can always be constructed from an arbitrary entangled state $|\psi\rangle$. See Table 4 for entropy, tangle, and Bell-state data for Figs. 1 and 2.

\section{APPENDIX C: PHYSICAL INPUT BASIS FOR PROCESS TOMOGRAPHY}

The basis operators $\hat{\rho}^{(j k)}$ we used for the process tomography were matrices with a single 1 in the jth row and $k$ th column. We can relate this to some other basis such as $\hat{\rho}^{(\alpha \beta)}=\hat{\rho}^{(\alpha)} \otimes \hat{\rho}^{(\beta)}$, where $\alpha, \beta \in\{H, V, D, R\}$. Clearly, it is trivial to write the $\hat{\rho}^{(\alpha \beta)}$ operators in terms of combinations of $\hat{\rho}^{(j k)}$. If we write this mapping as a matrix $\vec{\rho}^{(\alpha \beta)}$ $=M \vec{\rho}^{(j k)}$, we can now simply invert the matrix $\vec{\rho}^{(j k)}$ $=M^{-1} \vec{\rho}^{(\alpha \beta)}$, i.e., where $a=(1+i) / 2$ : 


$$
\left[\begin{array}{l}
\rho^{(11)} \\
\rho^{(12)} \\
\rho^{(13)} \\
\rho^{(14)} \\
\rho^{(21)} \\
\rho^{(22)} \\
\rho^{(23)} \\
\rho^{(24)} \\
\rho^{(31)} \\
\rho^{(32)} \\
\rho^{(33)} \\
\rho^{(34)} \\
\rho^{(41)} \\
\rho^{(42)} \\
\rho^{(43)} \\
\rho^{(44)}
\end{array}\right]\left[\begin{array}{cccccccccccccccc}
1 & 0 & 0 & 0 & 0 & 0 & 0 & 0 & 0 & 0 & 0 & 0 & 0 & 0 & 0 & 0 \\
-a & -a & 1 & i & 0 & 0 & 0 & 0 & 0 & 0 & 0 & 0 & 0 & 0 & 0 & 0 \\
-a & 0 & 0 & 0 & -a & 0 & 0 & 0 & 1 & 0 & 0 & 0 & i & 0 & 0 & 0 \\
i / 2 & i / 2 & -a & a^{*} & i / 2 & i / 2 & -a & a^{*} & -a & -a & 1 & i & a^{*} & a^{*} & i & -1 \\
-a^{*} & -a^{*} & 1 & -i & 0 & 0 & 0 & 0 & 0 & 0 & 0 & 0 & 0 & 0 & 0 & 0 \\
0 & 1 & 0 & 0 & 0 & 0 & 0 & 0 & 0 & 0 & 0 & 0 & 0 & 0 & 0 & 0 \\
1 / 2 & 1 / 2 & -a & -a^{*} & 1 / 2 & 1 / 2 & -a & -a^{*} & -a^{*} & -a^{*} & 1 & -i & -a & -a & i & 1 \\
0 & -a & 0 & 0 & 0 & -a & 0 & 0 & 0 & 1 & 0 & 0 & 0 & i & 0 & 0 \\
-a^{*} & 0 & 0 & 0 & -a^{*} & 0 & 0 & 0 & 1 & 0 & 0 & 0 & -i & 0 & 0 & 0 \\
1 / 2 & 1 / 2 & -a^{*} & -a & 1 / 2 & 1 / 2 & -a^{*} & -a & -a & -a & 1 & i & -a^{*} & -a^{*} & -i & 1 \\
0 & 0 & 0 & 0 & 1 & 0 & 0 & 0 & 0 & 0 & 0 & 0 & 0 & 0 & 0 & 0 \\
0 & 0 & 0 & 0 & -a & -a & 1 & i & 0 & 0 & 0 & 0 & 0 & 0 & 0 & 0 \\
i / 2 & i / 2 & -a^{*} & a & i / 2 & i / 2 & -a^{*} & a & -a^{*} & -a^{*} & 1 & -i & a & a & -i & -1 \\
0 & -a^{*} & 0 & 0 & 0 & -a^{*} & 0 & 0 & 0 & 1 & 0 & 0 & 0 & -i & 0 & 0 \\
0 & 0 & 0 & 0 & -a^{*} & -a^{*} & 1 & -i & 0 & 0 & 0 & 0 & 0 & 0 & 0 & 0 \\
\rho^{(H R)} \\
\rho^{(V H)} \\
\rho^{(V V)} \\
\rho^{(V D)} \\
\rho^{(V R)} \\
\rho^{(D H)} \\
\rho^{(D V)} \\
\rho^{(D D)} \\
\rho^{(D R)} \\
\rho^{(R H)} \\
\rho^{(R V)} \\
\rho^{(R D)} \\
\rho^{(R R)}
\end{array}\right]
$$

\section{APPENDIX D: PROCESS TOMOGRAPHY OF A TWO-QUBIT GATE-AN EXAMPLE}

First we need a basis of input states. For each qubit, use the following input states:

$$
\begin{array}{ll}
\hat{\rho}^{(H)}=\left[\begin{array}{ll}
1 & 0 \\
0 & 0
\end{array}\right], & \hat{\rho}^{(V)}=\left[\begin{array}{ll}
0 & 0 \\
0 & 1
\end{array}\right], \\
\hat{\rho}^{(D)}=\frac{1}{2}\left[\begin{array}{ll}
1 & 1 \\
1 & 1
\end{array}\right], & \hat{\rho}^{(R)}=\frac{1}{2}\left[\begin{array}{cc}
1 & -i \\
i & 1
\end{array}\right],
\end{array}
$$

so that the basis for the two qubits comprises 16 twoqubit states given by all the tensor products of the single qubit states $\left\{\hat{\boldsymbol{\rho}}^{(\alpha \beta)}\right\}=\left\{\hat{\boldsymbol{\rho}}^{(\alpha)} \otimes \hat{\boldsymbol{\rho}}^{(\beta)}\right\}, \alpha, \beta \in\{H, V, D, R\}$. For example,

$$
\hat{\rho}^{(D R)}=\frac{1}{4}\left[\begin{array}{cccc}
1 & -i & 1 & -i \\
i & 1 & i & 1 \\
1 & -i & 1 & -i \\
i & 1 & i & 1
\end{array}\right] .
$$

Using $\left\{\hat{\boldsymbol{\rho}}^{(\alpha \beta)}\right\}$ as input states and performing state tomography on each one, we obtain a set of output matrices $\left\{\mathcal{E}\left(\hat{\rho}^{(\alpha \beta)}\right)\right\}$.

Using the transformation in Appendix C, we can easily calculate the $16\left\{\mathcal{E}\left(\hat{\rho}^{(j k)}\right)\right\}$ matrices we need to construct the $\chi$-matrix in Eq. (23). For example, with $a=(1+i) / 2$,

$$
\mathcal{E}\left(\hat{\rho}^{(13)}\right)=-a \mathcal{E}\left(\hat{\rho}^{(H H)}\right)-a \mathcal{E}\left(\hat{\rho}^{(V H)}\right)+\mathcal{E}\left(\hat{\rho}^{(D H)}\right)+i \mathcal{E}\left(\rho^{(R H)}\right) .
$$

For the purposes of illustration, let us consider the following process, which applies a CNOT gate with probability $p$ else does nothing,

$$
\mathcal{E}(\hat{\rho})=p \operatorname{CNOT} \hat{\rho} \mathrm{CNOT}+(1-p) \hat{\rho},
$$

and imagine that we obtain the output state $\mathcal{E}(\hat{\rho})$ by state tomography. Constructing the $\chi$-matrix in block form as given in Eq. (23), we arrive at

$$
\chi=\frac{1}{4}\left[\begin{array}{ccccccccc}
4-3 p & p & 0 & & 0 & p & -p & 0 & 0 \\
p & p & 0 & \cdots & 0 & p & -p & 0 & 0 \\
0 & 0 & 0 & & 0 & 0 & 0 & 0 & 0 \\
& \vdots & & & & & \vdots & & \\
0 & 0 & 0 & & 0 & 0 & 0 & 0 & 0 \\
p & p & 0 & & 0 & p & -p & 0 & 0 \\
-p & -p & 0 & \cdots & 0 & -p & p & 0 & 0 \\
0 & 0 & 0 & & 0 & 0 & 0 & 0 & 0 \\
0 & 0 & 0 & & 0 & 0 & 0 & 0 & 0
\end{array}\right] .
$$

It is fairly easy to read the $\chi$-matrix directly-consider the extreme cases of $p=0$ and $p=1$. For $p=0$ only the top right element is nonzero, and this corresponds to $I \otimes I$; i.e., no transformation is applied. For $p=1$, writing out the element from the matrix according to Eq. (20) and factorizing, $\mathcal{E}(\hat{\rho})=U \hat{\rho} U^{\dagger}$, where $U=\frac{1}{2}(I \otimes I+I \otimes X+Z \otimes I-Z \otimes X)$ and it is readily verified that $U=\mathrm{CNOT}$.

\section{ACKNOWLEDGMENTS}

We acknowledge J. B. Altepeter, S. D. Bartlett, C. M. Dawson, D. F. V. James, D. Mortimer, W. J. Munro, and M. A. Nielsen for invaluable discussions. This work was partially supported by the Australian Research Council and by the U.S. Disruptive Technologies Office.

Corresponding author A. White can be reached by e-mail at andrew.white@uq.edu.au. 


\section{REFERENCES AND NOTES}

1. M. A. Nielsen and I. L. Chuang, Quantum Computation and Quantum Information (Cambridge U. Press, 2000).

2. J. L. Brylinski and R. K. Brylinski, "Computational mathematics," in Mathematics of Quantum Computation, R. K. Brylinski and G. Chen, eds. (Chapman \& Hall/CRC Press, 2002), Chap. 2.

3. M. J. Bremner, C. M. Dawson, J. L. Dodd, A. Gilchrist, A. W. Harrow, D. Mortimer, M. A. Nielsen, and T. J. Osborne, "Practical scheme for quantum computation with any twoqubit entangling gate," Phys. Rev. Lett. 89, 247902 (2002).

4. R. J. Hughes, ed., "A quantum information science and technology roadmap," Rep. LA-UR-04-1778, ARDA, April 2, 2004, http://qist.lanl.gov/.

5. F. Schmidt-Kaler, H. Häffner, M. Riebe, S. Gulde, G. P. T. Lancaster, T. Deuschle, C. Becher, C. F. Roos, J. Eschner and R. Blatt, "Realization of the Cirac-Zoller controlledNOT quantum gate," Nature 422, 408-411 (2003).

6. J. L. O'Brien, G. J. Pryde, A. G. White, T. C. Ralph, and D. Branning, "Demonstration of an all-optical quantum controlled-NOT gate," Nature 426, 264-267 (2003).

7. A. G. White, D. F. V. James, P. H. Eberhard, and P. G. Kwiat, "Nonmaximally entangled states: production, characterization, and utilization," Phys. Rev. Lett. 83, 3103-3107 (1999).

8. On the qubit characteristic sphere the equal-weight superpositions form a great circle midway between the logical states. For example, on the Bloch sphere they lie on the equator, which can be accessed with $\pi / 2$ pulses; on the Poincare sphere they lie on the great circle containing the diagonally and circularly polarized states.

9. C. A. Sackett, D. Kielpinski, B. E. King, C. Langer, V. Meyer, C. J. Myatt, M. Rowe, Q. A. Turchette, W. M. Itano, D. J. Wineland, and C. Monroe, "Experimental entanglement of four particles," Nature 404, 256-259 (2000).

10. B. M. Terhal and P. Horodecki, "Schmidt number for density matrices," Phys. Rev. A 61, 040301(R) (2000).

11. A. Gilchrist, N. K. Langford, and M. A. Nielsen, "Distance measures to compare real and ideal quantum processes," Phys. Rev. A 71, 062310 (2005).

12. J. L. Dodd and M. A. Nielsen, "A simple operational interpretation of the fidelity," Phys. Rev. A 66, 044301 (2002)

13. M. Steffen, M. Ansmann, R. C. Bialczak, N. Katz, E. Lucero, R. McDermott, M. Neeley, E. M. Weig, A. N. Cleland, and J. M. Martinis, "Measurement of the entanglement of two superconducting qubits via state tomography," Science 313, 1423-1425 (2006)

14. R. Alicki, "False qubits II. Entanglement of Josephson junctions," arXiv.org e-Print archive, quantum physics/ 0609122, 16 September 2006, http://www.arxiv.org/abs/ quant-ph/0609122.

15. M. Horodecki, P. Horodecki, and R. Horodecki, "Separability of mixed states: necessary and sufficient conditions," Phys. Lett. A 223, 1-8 (1996).

16. B. M. Terhal, "Bell inequalities and the separability criterion," Phys. Lett. A 271, 319-326 (2000).

17. M. Barbieri, F. De Martini, G. Di Nepi, P. Mataloni, G. M. D'Ariano, and C. Macchiavello, "Detection of entanglement with polarized photons: experimental realization of an entanglement witness," Phys. Rev. Lett. 91, 227901 (2003).

18. O. Gühne, P. Hyllus, D. Bruß, A. Ekert, M. Lewenstein, C. Macchiavello, and A. Sanpera, "Detection of entanglement with few local measurements," Phys. Rev. A 66, 062305 (2002).

19. The partial transpose of $\hat{\rho}$, with respect to an orthonormal basis $\{|b\rangle\}$ of qubit two, is given by $\hat{\rho}^{T_{2}}=\Sigma_{b b^{\prime}}\left|b^{\prime}\right\rangle\left\langle b|\hat{\rho}| b^{\prime}\right\rangle\langle b|$.

20. D. F. V. James, P. G. Kwiat, W. J. Munro, and A. G. White, "Measurement of qubits," Phys. Rev. A 64, 052312 (2001).

21. A. Doherty and A. Gilchrist, Department of Physics, University of Queensland, Brisbane QLD 4072, Australia are preparing a manuscript to be called "Quantum tomography using convex optimisation."
22. R. Blume-Kohout and P. Hayden, "Accurate quantum state estimation via 'Keeping the experimentalist honest'," arXiv.org e-Print archive, quantum physics/0603116, 14 March 2006, http://www.arxiv.org/abs/quant-ph/0603116.

23. A. G. White, D. F. V. James, W. J. Munro, and P. G. Kwiat, "Exploring Hilbert space: accurate characterization of quantum information," Phys. Rev. A 65, 012301 (2001).

24. W. K. Wootters, "Entanglement of formation of an arbitrary state of two qubits," Phys. Rev. Lett. 80, 2245-2248 (1998).

25. V. Coffman, J. Kundu, and W. K. Wootters, "Distributed entanglement," Phys. Rev. A 61, 052306 (2000).

26. W. J. Munro, K. Nemoto, and A. G. White, "The Bell inequality: a measure of entanglement?" J. Mod. Opt. 48, 1239-1246 (2001).

27. W. J. Munro, D. F. V. James, A. G. White, and P. G. Kwiat, "Maximizing the entanglement of two mixed qubits," Phys. Rev. A 64, 030302 (2001).

28. N. A. Peters, J. B. Altepeter, D. A. Branning, E. R. Jeffrey, T.-C. Wei, and P. G. Kwiat, "Maximally entangled mixed states: creation and concentration," Phys. Rev. Lett. 92, 133601 (2004)

29. Assuming that count statistics were the dominant source of uncertainty, 1000 Poissonian-distributed data sets (and hence reconstructed density matrices) were generated from each observed data set. The tangle, entropy, and fidelity were calculated for all 1000, yielding the standard deviation of each measure about the mean.

30. J. S. Bell, "On the Einstein-Podolsky-Rosen paradox," Physics (Long Island City, N.Y.) 1, 195-200 (1964).

31. J. F. Clauser, M. A. Horne, A. Shimony, and R. A. Holt, "Proposed experiment to test local hidden-variable theories," Phys. Rev. Lett. 23, 880-884 (1969).

32. To be conclusive in this regard, Bell's inequality requires high-efficiency spacelike separated measurements, a feat not yet achieved in any experimental architecture.

33. Note that an operator sum representation is not unique and that there is unitary freedom in its choice.

34. J. B. Altepeter, D. Branning, E. Jeffrey, T. C. Wei, P. G. Kwiat, R. T. Thew, J. L. OBrien, M. A. Nielsen, and A. G. White, "Ancilla-assisted quantum process tomography," Phys. Rev. Lett. 93, 193601 (2003).

35. G. M. D'Ariano and P. L. Presti, "Imprinting a complete information about a quantum channel on its output state," Phys. Rev. Lett. 91, 047902 (2003).

36. F. De Martini, A. Mazzei, M. Ricci, and G. M. D’Ariano, "Exploiting quantum parallelism of entanglement for a complete experimental quantum characterization of a single qubit device," Phys. Rev. A 67, 062307 (2003).

37. I. L. Chuang and M. A. Nielsen, "Prescription for experimental determination of the dynamics of a quantum black box," J. Mod. Opt. 44, 732-744 (1997).

38. J. F. Poyatos, J. I. Cirac, and P. Zoller, "Complete characterization of a quantum process: the two-bit quantum gate," Phys. Rev. Lett. 78, 390-393 (1997).

39. M. A. Nielsen, E. Knill, and R. Laflamme, "Complete quantum teleportation using nuclear magnetic resonance," Nature 396, 52-55 (1998).

40. D. W. Leung, "Towards robust quantum computation," arXiv.org e-Print archive, computer science/10012017, 20 December 2002, http://arxiv.org/abs/cs.CC/0012017.

41. A. M. Childs, I. L. Chuang, and D. W. Leung, "Realization of quantum process tomography in NMR," Phys. Rev. A 64, 012314 (2001).

42. J. L. O’Brien, G. J. Pryde, A. Gilchrist, D. F. V. James, N. K. Langford, T. C. Ralph, and A. G. White, "Quantum process tomography of a controlled-NOT gate," Phys. Rev. Lett. 93, 080502 (2004).

43. M. Mohseni and D. A. Lidar, "Direct characterization of quantum dynamics: I. General theory," arXiv.org e-Print archive, quantum physics/0601033, 5 January 2006, http:// www.arxiv.org/abs/quant-ph/0601033.

44. M. Ziman, "Notes on optimality of direct characterisation of quantum dynamics," arXiv.org e-Print archive, quantum physics/0603151, 17 March 2006, http://www.arxiv.org/abs/ quant-ph/0603151.

45. N. K. Langford, T. J. Weinhold, R. Prevedel, A. Gilchrist, J. 
L. O'Brien, G. J. Pryde, and A. G. White, "Demonstration of a simple entangling optical gate and its use in Bell-state analysis," Phys. Rev. Lett. 95, 210504 (2005).

46. A. Jamiolkowski, "Linear transformations which preserve trace and positive semidefiniteness of operators," Rep. Math. Phys. 3, 275-278 (1972).

47. J. I. Cirac, W. Dür, B. Kraus, and M. Lewenstein, "Entangling operations and their implementation using a small amount of entanglement," Phys. Rev. Lett. 86, 544-547 (2001).

48. M. Horodecki, P. Horodecki, and R. Horodecki, "General teleportation channel, singlet fraction, and quasidistillation," Phys. Rev. A 60, 1888-1898 (1999).
49. M. A. Nielsen, "A simple formula for the average gate fidelity of a quantum dynamical operation," Phys. Lett. A 303, 249-252 (2002).

50. H. F. Hofmann, "Complementary classical fidelities as an efficient criterion for the evaluation of experimentally realized quantum operations," Phys. Rev. Lett. 94, 160504 (2005).

51. H. F. Hofmann, R. Okamoto, and S. Takeuchi, "Analysis of an experimental quantum logic gate by complementary classical operations," arXiv.org e-Print archive, quantum physics/0608005, 1 August 2006, http://www.arxiv.org/abs/ quant-ph/0608005. 